Published in final edited form as:

Clin Gastroenterol Hepatol. 2012 July ; 10(7): 786-794. doi:10.1016/j.cgh.2012.01.020.

\title{
Association between Puberty and Features of Nonalcoholic Fatty Liver Disease
}

\author{
Ayako Suzuki'), Manal F. Abdelmalek1), Jeffrey B. Schwimmer ${ }^{2), 3)}$, Joel E. Lavine ${ }^{4)}$, Ann \\ Scheimann $^{5}$, Aynur Unalp-Arida ${ }^{6)}$, Katherine Yates $^{6)}$, Arun Sanyal ${ }^{7)}$, Cynthia D Guy ${ }^{8}$, and \\ Anna Mae Dieh ${ }^{1}{ }^{1}$ for the NASH CRN \\ 1)Division of Gastroenterology, Duke University, Durham, NC \\ 2)Department of Pediatrics, University of California San Diego, La Jolla, CA \\ 3)Department of Gastroenterology, Rady Children's Hospital, San Diego, CA \\ 4)Division of Pediatric Gastroenterology and Nutrition, Columbia College of Physicians and \\ Surgeons, New York, NY \\ 5)Pediatric Gastroenterology, Johns Hopkins Children's Center, Baltimore, MD \\ 6)NASH CRN Data Coordinating Center, Johns Hopkins Bloomberg School of Public Health, \\ Baltimore, MD \\ 7)Division of Gastroenterology, Virginia Commonwealth University, Richmond, VA \\ 8)Department of Pathology, Duke University, Durham, NC
}

\section{Abstract}

Background \& Aims-Physiologic changes that occur during puberty may affect pathologic features of nonalcoholic fatty liver disease (NAFLD). We investigated associations between pubertal development and clinical and histopathologic features of NAFLD.

Methods-We studied 186 children (age<18 years, 143 boys) with biopsy-proven NAFLD. The population was divided into 3 groups, based on Tanner stage (pre-puberty, puberty, and postpuberty). Clinical characteristics and histologic features were compared among groups. Multivariable regression models were used to adjust for potential confounders.

(C) 2012 The American Gastroenterological Association. Published by Elsevier Inc. All rights reserved.

Corresponding author: Ayako Suzuki, M.D., Ph.D., M.Sc., Duke University Medical Center, Division of Gastroenterology, Duke South Orange Zone, Room 0340, PO 3913, Durham, NC, 27710, TEL: 919-668-8891, FAX: 919-681-8147, suzuk004@ mc.duke.edu. Conflict of Interest: The authors report no conflict of interest.

Contribution: Ayako Suzuki contributed to study concept and design, statistical analysis, interpretation of data and drafting of the manuscript; Manal F. Abdelmalek contributed to interpretation of data and critical revision of the manuscript for important intellectual content; Jeffrey Schwimmer contributed to study design, acquisition of data, interpretation of data, and critical revision of the manuscript for important intellectual content; Joel Lavine and Ann Scheimann contributed to acquisition of data and critical revision of the manuscript for important intellectual content; Aynur Unalp-Arida contributed to acquisition of data and critical revision of the manuscript for important intellectual content; Katherine Yates contributed to statistical analysis and critical revision of the manuscript for important intellectual content; Arun Sanyal contributed to critical revision of the manuscript for important intellectual content; Cynthia D Guy contributed to generation of histologic data, analysis and interpretation of data and critical revision of the manuscript for important intellectual content; Anna Mae Diehl contributed to critical revision of the manuscript for important intellectual content.

Publisher's Disclaimer: This is a PDF file of an unedited manuscript that has been accepted for publication. As a service to our customers we are providing this early version of the manuscript. The manuscript will undergo copyediting, typesetting, and review of the resulting proof before it is published in its final citable form. Please note that during the production process errors may be discovered which could affect the content, and all legal disclaimers that apply to the journal pertain. 
Results-After adjusting for other factors, hyperuricemia and low levels of high-density lipoprotein cholesterol were more prevalent among children who entered puberty with lower levels of quantitative insulin-sensitivity check index $(\mathrm{P}<.05)$. The degree of steatosis, numbers of Mallory-Denk bodies, and diagnostic categories of NAFLD differed among groups $(\mathrm{P}<.05)$. There were potential sex differences in associations between stages of puberty and lobular inflammation, hepatocyte ballooning, and borderline steatohepatitis of zone 3; these were therefore not included in multivariable analyses of the overall population. Following adjustment for different sets of confounders, patients at or beyond puberty were less likely to have high-grade steatosis, severe portal inflammation, borderline steatohepatitis (zone 1), or a high stage of fibrosis than patients who had not entered puberty $(\mathrm{P}<.05)$. On the contrary, the prevalence of Mallory-Denk body was greater among post-puberty subjects $(P=.06)$.

Conclusion-Steatosis, portal inflammation, and fibrosis are less severe during or after puberty than before puberty among subjects with NAFLD. Post-pubescent individuals have a lower prevalence of borderline steatohepatitis of zone 1 but are more likely to have Mallory-Denk bodies. These findings indicate that puberty affects the pathologic features of NAFLD.

\section{Keywords}

sexual development; nonalcoholic steatohepatitis; obesity; sex hormones; QUICKI

\section{INTRODUCTION}

Nonalcoholic fatty liver disease (NAFLD) is a significant public health problem in industrialized nations even among children. ${ }^{1,2}$ The prevalence of NAFLD among children is $9.6 \%$ as estimated in a previous autopsy study. ${ }^{2}$ Advanced forms of NAFLD (e.g., cirrhosis) have been also reported among children. ${ }^{3}$ Histologic features observed among children are often different from those observed in adults. ${ }^{4} 5$ Potential mechanisms explaining the distinct histologic features observed in pediatric NAFLD have not been fully investigated and no plausible explanation is currently available. Whether physiological alterations associated with gender or the reproductive lifecycle (e.g., puberty, pregnancy, or menopause) impact disease development or progression remains unclear.

The development of NAFLD in adult populations appears to be influenced by gender as well as reproductive state. ${ }^{6}$ Several population-based studies consistently show that the prevalence of fatty liver is higher in men compared to women during their reproductive age. ${ }^{6}$ Among adolescents, there is an increased prevalence of fatty liver in boys compared to girls. ${ }^{2,6}$ On the other hand, after menopause (or after 50 years), the prevalence of fatty liver among women exceeds that of men. ${ }^{6}$ In a cohort study done in Japan, male gender was associated with increased hazard ratio of incident hypertransaminasemia before 40 year old, while after 40 years old the gender difference was eliminated. ${ }^{7}$ The prevalence of hypertransaminasemia is also influenced by hormone-replacement therapy among postmenopausal women. ${ }^{8}$ Thus, the gender difference is likely due to difference(s) in sex hormones. Currently, limited data are available to assess impact of gender and reproductive state on the disease progression of NAFLD. The question of whether gender impacts the disease progression of NAFLD, and if so how, remains unanswered. Our recent analysis using adult NAFLD patients showed that the prevalence of advanced fibrosis is higher in post-menopausal women than in pre-menopausal women and men. ${ }^{9}$ Further, in chronic viral hepatitis, estrogen exposure appears to be protective against fibrosis progression while menopause is detrimental. ${ }^{10}$ Thus, it is plausible that gender, reproductive state, pregnancy, oral contraceptives, and hormone replacement therapy influence the development and, probably, severity of NAFLD in adults. 
How gender and puberty influence the development and the severity of NAFLD has not been fully investigated. Previous studies showed that NAFLD is more prevalent among boys ${ }^{11}$ and boys are more likely to have type 2 (pediatric/peri-portal/zone 1 pattern) nonalcoholic steatohepatitis (NASH). ${ }^{5}$ Another study showed that mean Tanner stage was univariately associated with different fibrosis stages as well as different types of NASH. ${ }^{12}$ However, the influence of pubertal development has not been fully assessed. Thus, whether pubertal development influences the severity of liver histology findings in NAFLD and, if so, whether the influence is gender-specific remains unknown. Sex hormones impact various physiological and biological functions, including adipocyte development and function. ${ }^{13}$ Further, insulin sensitivity is decreased during puberty for unknown reasons. ${ }^{14}$ Thus, physiological changes associated with puberty (e.g., rise in sex hormone levels, decreased insulin sensitivity) may significantly impact disease manifestations of NAFLD.

To better understand potential influences of gender and reproductive lifecycle on histologic features, we analyzed a pediatric cohort with NAFLD in collaboration with the Nonalcoholic Steatohepatitis Clinical Research Network (NASH CRN). We also analyzed regional anthropometric measures and clinical features of insulin resistance along with pubertal development, because these may be altered during puberty and thus may impact histologic severity. Our specific aims were to assess the association of pubertal stages (pre-puberty, puberty, and post-puberty) with regional anthropometric measures, clinical features of insulin resistance and severity of histologic features of NAFLD with consideration of potential gender differences.

\section{METHODS}

\section{Study design and population}

We performed a cross-sectional analysis using data from the NASH CRN Database study and the TONIC (Treatment of NAFLD in Children) clinical trial. The study design and data collected from the NAFLD Database study and TONIC trial have been reported recently. ${ }^{15,16}$ Our study population required the following criteria: 1) age at enrollment was $<18$ years, 2) the presence of liver histologic data, 3) no chronic liver diseases other than NAFLD as assessed by serologies and/or histology, and 4) the presence of study variables obtained at baseline and within 3 months of liver biopsy. The NASH CRN studies were approved by the Institutional Review Boards at each center.

\section{Liver histology}

The primary outcome in this study was the severity of each histologic feature: steatosis, lobular inflammation, portal inflammation, Mallory-Denk bodies, hepatocyte ballooning, and fibrosis. To characterize associations between pubertal stages and patterns of inflammation, the diagnostic categorization [simple steatosis/no steatohepatitis; borderline steatohepatitis zone 3 (adult pattern); borderline steatohepatitis zone 1 (pediatric or periportal pattern); definite steatohepatitis] ${ }^{12,17}$ was also analyzed. NASH CRN scoring criteria does not distinguish adult vs. pediatric patterns within the diagnostic category of definite steatohepatitis; therefore, definite steatohepatitis was classified as one category regardless of injury patterns. ${ }^{12,17}$ All liver biopsies from the enrolled patients were stained with hematoxylin-eosin and Masson's trichrome stains, and reviewed and scored centrally by the NASH CRN Pathology Committee periodically convened since 2005 in a consensus manner, according to the published scoring system. ${ }^{17,18}$ For the analyses, some grades/ stages of the histologic features were combined as follows: steatosis (3 levels: grade $0-1,2$ and 3), lobular inflammation ( 2 levels: grade $0-1$ vs. $2-3$ ), portal inflammation ( 2 levels: grade $0-1$ vs. 2 ), Mallory-Denk bodies ( 2 levels: yes vs. no), hepatocyte ballooning (2 levels: grade $0-1$ vs. 2 ), and fibrosis (4 levels: stage $0,1,2$, and 3-4). 


\section{Study Variables}

Pubertal development-The variable of pubertal development was the primary predictor in this study. Based on Tanner stages (Table 1), ${ }^{19,} 20$ pubertal development was classified into three stages, pre-puberty, puberty, and post-puberty. For girls, Tanner stages for pubic hair and breasts were used. ${ }^{19}$ Pre-puberty was defined as both breast and genital Tanner stages being stage 1 , while post-puberty was defined as both Tanner stages being stage 5 . The remainder of the cases were classified into puberty. In cases where only one component of Tanner stages (pubic hair or breasts) was available, then the case was classified based on the available Tanner stage component. For boys, Tanner stages for pubic hair and genitals were used to classify the stages of pubertal development using the same manner. ${ }^{20}$

Other study variables-Demographic information, anthropometric measures [height $(\mathrm{cm})$, weight $(\mathrm{kg})$, body mass index $\left(\mathrm{BMI}, \mathrm{kg} / \mathrm{m}^{2}\right)$, the circumferences of waist (WAIST, $\mathrm{cm}$ ), hip (HIP, $\mathrm{cm}$ ) and mid-upper arm (ARM, cm), and triceps skinfold $(\mathrm{cm})]$, physical activity, the presence or absence of clinical features of insulin resistance, and quantitative insulin sensitivity check index (QUICKI) were collected at the time of the study enrollment (within 3 months from liver biopsy) via case report forms developed by the NASH CRN Steering Committee. Individual clinical features of insulin resistance were defined as follows: diabetes or impaired fasting glucose (IFG) (patient report and/or use of antidiabetics and/or fasting glucose $>100 \mathrm{~g} / \mathrm{dl}$ ), hypertension (patient report and/or use of antihypertensives), hyperuricemia (serum uric acid $>5.5 \mathrm{mg} / \mathrm{dl}$ ), hypertriglyceridemia (serum triglycerides $>150 \mathrm{mg} / \mathrm{dl}$ ), elevated LDL-cholesterol (serum LDL-cholesterol $\geq 130 \mathrm{mg} / \mathrm{dl}$ ), low HDL-cholesterol (serum HDL-cholesterol $<40 \mathrm{mg} / \mathrm{dl}$ in boys and $<50 \mathrm{mg} / \mathrm{dl}$ in girls). For race and ethnicity, we used a combination variable [White (non-Hispanic), Hispanic, and others]. Total caloric intake (Kcal/day) was calculated based on self-reported usual eating habits over the prior year provided via the Block Brief Questionnaire. ${ }^{21}$ Four variables for physical activity levels were created using available self-reported information as follows (modifiable activity questionnaire): 1) recreational physical activities (METhours/week) calculated based on all the reported recreational activities (at least 10 times) during the past year (i.e., recreational activity), 2) any exercise (more than $20 \mathrm{~min}$, either light or hard exercise) more than 2 days a week during the recent 2 weeks (yes or no) (i.e., recent regular exercise), 3) participation in competitive sports during the recent 2 weeks (yes or no), and 4) TV or computer time during the recent 2 weeks (more than 4 hours per day, yes or no) (i.e., sedentary activity).

\section{Statistical analyses}

Data are reported as mean $\pm \mathrm{SD}$ for continuous variables or proportion of patients with a condition. We first compared clinical characteristics (including anthropometric measures and clinical features of insulin resistance) among the three groups: pre-puberty, puberty, and post-puberty by using ANOVA, Chi-square tests or exact tests. For the comparison of anthropometric measures across age groups and sexes, z-scores were also used. ${ }^{22}$ Multiple linear (or logistic) regression models were used to assess the associations between pubertal stages and clinical features of insulin resistance after adjusting for other confounding factors.

We then assessed associations between the pubertal stages and severity of each histologic feature in the total study population using Chi-Square tests or exact tests. To assess potential gender interaction, ordinal logistic (or logistic) regression analysis was performed for each histologic feature including interaction term (gender * pubertal stages). For the diagnostic categories, multinomial logistic regression was used for this purpose. For histologic features that did not show apparent gender difference, we performed multiple ordinal logistic (or logistic) regression analyses in the total study population to assess the associations between 
pubertal stages and the severity of histologic features after adjusting for different sets of confounding factors: 1) Model 1: gender, ethnicity, race, calorie intake, physical activity levels, height (z-score), and weight (z-score) and 2) Model 2: Model $1+$ hypertension, diabetes/impaired glucose tolerance, and hypertriglyceridemia, and QUICKI. For binary count outcome data and categorical factors of interest, in situations when a categorical factor has a category with 0 outcome counts, maximum likelihood estimate of the factor effect in the logistic regression model does not exist ("separation" problem). In such cases we either used an altered factor classification in that particular model or did not report the estimates with explanation provided.

Adjusted cumulative odds ratios (ACOR) or odds ratio (AOR) of individual pubertal stages were estimated from each model using 'pre-puberty' or a combination of pre-puberty and puberty as a reference group. In a separate analysis, we performed the models using age, instead of the pubertal stages, and assessed the associations with histologic severity. $P$ values were determined from a likelihood ratio test.

For analyses, we used JMP statistical software version 8.0 (SAS institute Inc., Cary, NC) and considered differences statistically significant when the p-values were less than 0.05 . All $P$ values presented are two-sided. Due to a descriptive nature of this analysis, $\mathrm{p}$-values have not been adjusted for multiple comparisons.

\section{RESULTS \\ Clinical Characteristics}

A total of 186 children (age < 18 years) with NAFLD were analyzed. Median and interquartile range (in months) of difference between the time of liver biopsy and the time of the clinical data collection was $2(1,2)$. Clinical characteristics of the patients were significantly different among the three pubertal stages (Table 2). As anticipated, pubertal development increased along with growth, age and all the anthropometric measures; the associations were statistically significant, except for triceps skin fold. After standardizing the anthropometric measures, BMI, waist circumference and hip circumferences were significantly different among the pubertal stages (ANOVA, $p<0.05$ ); pre-puberty was associated with the highest $\mathrm{z}$-scores of BMI, waist circumference and hip circumference (data are not shown). Gender ( $\mathrm{p}=0.02)$, race-ethnicity $(\mathrm{p}=0.04)$, and competitive sport activities $(\mathrm{p}=0.02)$ were also significantly different among the three stages.

With respect to clinical features of insulin resistance, hypertriglyceridemia ( $\mathrm{p}=0.03)$, low HDL-cholesterol $(\mathrm{p}=0.06)$, and hyperuricemia $(\mathrm{p}<0.0001)$ became more prevalent after the onset of puberty (Table 2). In logistic regression models adjusting for gender, race-ethnicity, standardized BMI (z-scores), and competitive sport activities, the pubertal stages were significantly associated with low HDL-cholesterol and hyperuricemia (likelihood ratio test: $\mathrm{p}=0.02$ and $\mathrm{p}<0.0001$, respectively). Further, in a multiple linear regression model including the same set of variables, QUICKI was significantly associated with the pubertal stages (effect test, $\mathrm{p}=0.003)$ and lower in puberty $(\beta=-0.017 \pm 0.006, \mathrm{p}=0.004)$ and post-puberty $(\beta=-0.024 \pm 0.008, \mathrm{p}=0.002)$ vs. pre-puberty.

\section{The associations between the stages of pubertal development and histologic severity of NAFLD}

We compared the severity of histologic features among the pubertal stages in the total population and assessed gender interaction (Table 3). The severity of steatosis and MalloryDenk bodies was significantly different among the pubertal stages (exact tests, $\mathrm{p}$ values $<0.05$ ) while severe portal inflammation tended to be associated with the pubertal stages (exact test, $\mathrm{p}=0.07$ ). In more advanced pubertal stages, lower grades of steatosis, less 
prevalent severe portal inflammation, and more prevalent Mallory-Denk bodies were noted (Figure 1). The diagnostic categories were also significantly different among the pubertal stages (exact test, $\mathrm{p}=0.005$ ) (Figure 1). Especially, borderline steatohepatitis zone 1 (pediatric pattern) was significantly more prevalent in earlier stages (pre-puberty vs. puberty vs. post-puberty $=35.4 \%$ vs. $20.0 \%$ vs. $3.2 \%$ test $)($ Table 3$)$.

Based on the data distributions in boys and girls, a potential gender difference was suggested in the relationship of pubertal stages with lobular inflammation, severe hepatocyte ballooning, and borderline steatohepatitis zone 3 (Table 3). Among all the histologic features, statistically significant gender interaction was detected only for lobular inflammation $(\mathrm{p}=0.01)$ while valid models could not be developed for portal inflammation, hepatocyte ballooning and Mallory-Denk body due to the 'separation' problem. The features that showed potential gender interaction in their distribution were excluded from the following multivariable analyses using overall population.

For histologic features that did not show apparent gender interaction, we assessed the associations with pubertal stages after adjusting for different sets of confounding factors. Unadjusted and adjusted ACORs/AORs of the pubertal stages for the each histologic feature are summarized in Table 4. After adjusting for gender, race-ethnicity, caloric intake, physical activity variables, body size (standardized height and weight), and insulin resistance-related variables, the severity of steatosis $(\mathrm{p}=0.005)$, portal inflammation $(\mathrm{p}=0.016)$, and fibrosis $(\mathrm{p}=0.040)$ and the diagnosis of borderline steatohepatitis zone 1 $(\mathrm{p}=0.006)$ were significantly associated with the pubertal stages. Overall, the stages of puberty and/or post-puberty were associated with a lower likelihood of these histologic features when compared to pre-puberty (Table 4, Model 2).

In contrast, the histologic finding of greater numbers of Mallory-Denk bodies was associated with post-pubertal stage $(\mathrm{p}=0.044)$. Even after adjusting for gender, race-ethnicity, caloric intake, physical activity variables, body size (standardized height and weight), and insulin resistance-related variables, post-puberty tended to be associated with a greater likelihood of having Mallory-Denk bodies ( $\mathrm{p}=0.06)$ (Table 4$)$.

Next, the models were developed replacing the pubertal stages with age. After adjusting for the confounders (using Model 2), older age was significantly associated with less severe steatosis $(\mathrm{p}=0.008)$, less severe portal inflammation $(\mathrm{p}=0.04)$, less severe fibrosis $(\mathrm{p}=0.0007)$, and a less prevalent diagnosis of borderline steatohepatitis zone $1(\mathrm{p}=0.0007)$, but was not associated with greater severity of Mallory-Denk bodies ( $\mathrm{p}=0.204)$; calculated ACOR or AOR for one year difference was $0.8[0.7,0.9]$ for steatosis, $0.7[0.5,1.0]$ for portal inflammation, $0.8[0.7,0.9]$ for fibrosis, and $0.7[0.5,0.8]$ for the diagnosis of borderline steatohepatitis zone 1 .

\section{DISCUSSION}

We conducted a cross-sectional analysis using the largest prospective pediatric NAFLD database and found that pubertal stages were associated with regional anthropometric measures, clinical features of insulin resistance, and the severity of several histologic features of NAFLD. Pubertal stages were univariately associated with the severity of steatosis, portal inflammation (borderline), Mallory-Denk bodies, and the diagnostic categories of steatohepatitis in the overall population. Children who were in their puberty and/or post-puberty at the time of study enrollment (within 3 months of liver biopsy) were less likely to have severe steatosis, severe portal inflammation, and the diagnosis of borderline steatohepatitis zone 1 (pediatric pattern), and were more likely to have increased numbers of Mallory-Denk bodies. After adjusting for different sets of confounders, the 
observed relationships were consistent (unadjusted vs. adjusted ORs). Furthermore, after the adjustment, post-puberty was associated with a decreased likelihood of having more severe fibrosis. Secondly, the associations of pubertal stages with hepatocyte ballooning, the diagnosis of borderline steatohepatitis zone 3 (adult pattern), and lobular inflammation appeared to be sexually dimorphic. Although we did not have enough statistical power to assess gender interaction in all the histologic features, lobular inflammation showed significant gender-interaction, suggesting that pubertal development may differentially impact, at least, certain histologic features in boys and girls.

Pubertal development causes physiological insulin resistance via as yet an unknown mechanism. After the onset of puberty, insulin sensitivity decreases, regardless of gender, ethnicity, and obesity, and returns to near pre-pubertal levels by the end of puberty. ${ }^{14}$ In our multivariable analyses, we observed a lower QUICKI and a greater prevalence of low HDLcholesterol and hyperuricemia in children who had experienced onset of puberty. This suggests that the observed differences in the parameters of insulin resistance cannot be explained by the differences in the other factors (i.e., gender, standardized BMI, race/ ethnicity, and physical activity) and may probably reflect physiological insulin resistance related to puberty. The adjustment for the parameters of insulin resistance, however, did not significantly alter the observed associations between pubertal stages and severity of histologic features (Table 4).

Potential mechanisms explaining the observed associations between the pubertal stages and the histologic features are unknown. Without assessing bio-physiological parameters that change over pubertal development, it is impossible to address underlying mechanisms. In our analyses, the associations were consistent regardless of adjusting for different sets of variables (i.e., demography, body size, calorie intake, physical activities, and parameters of insulin resistance), suggesting that the observed associations are independent of these factors. Sex hormone production dramatically increases throughout pubertal development. Thus, it is plausible to theorize that sex hormones may directly or indirectly impact pathologic features of NAFLD. A recent animal study showed that estrogen diminishes detrimental effects on mitochondrial function, ER stress markers, and free iron levels in the liver following shock injury. ${ }^{23}$ Another study reported that gender and estrogen influence Mallory-Denk body formation and oxidative stress in a mouse model of liver injury. ${ }^{24}$ Further, a previous animal study suggests that estrogens inhibit hepatic stellate cell activation and fibrogenesis. ${ }^{25}$ These findings in animal experiments suggest that gender and/ or sex hormones could impact cellular injury responses and modulate liver disease progression. Since serum estrogen levels rise both in boys and girls along with pubertal development ${ }^{26}$, it is possible that some of the observed associations may be explained by the alternation of estrogen levels during pubertal development. In this study, some histologic features suggested potential gender-specific relationship to pubertal stages, which indirectly supports the idea that some of the observed associations may be explained by differences in sex hormones. It is still inconclusive whether pubertal changes in sex hormones or other age-related factors influence the disease severity of NAFLD. Further investigation is warranted to better characterize influences of gender and reproductive lifecycle on NAFLD severity in a longitudinal study and to delineate how sex and sex hormones modulate mechanisms involved in injury and repair responses.

We recognize certain limitations in this study. First, we did not directly measure serum sex hormones or other bio-physiological alterations occurring across puberty (e.g., regional fat mass/distribution, lean body mass, metabolic rate, and other endocrinological changes).

Since the pubertal staging based on physical examination does not necessarily reflect sex hormone levels, ${ }^{27}$ the pubertal classification used in this study may not be optimal to address impact of sex hormones on the disease severity of NAFLD. Second, we did not 
assess onset of puberty or patterns of pubertal development (thelarche vs. adrenarche pathway) among girls. The patterns of development have been associated with anthropometric changes among girls. ${ }^{28}$ Thus, it is likely that the patterns may also impact disease severity of NAFLD. Further, oral contraceptive use likely alters sex hormone levels and thus could impact histologic features. As only 2 girls had received oral contraceptives in this population, this potential impact could not be assessed in the current study. Third, the cross-sectional nature of this study precludes us from addressing any questions pertaining to causality. Further duration of the disease or obesity was not taken into account in this study, which could have confounded some of the findings. Forth, the sample size and the data 'separation' precluded us from further characterization of gender interaction. Fifth, our findings may have been influenced by selection/referral bias as the study population was based on a cohort recruited from subspecialty centers around the country. Especially, about $60 \%$ was Hispanic in our population. Although we took into consideration race/ethnicity in our analysis, generalization of our findings requires extra caution prior to an independent validation.

In summary, this study revealed that pubertal stages were significantly associated with clinical features of insulin resistance and the severity of some histologic features in children with NAFLD. Potential gender differences were also noted in the relationship of some histologic features with the pubertal stages. Our findings suggest that puberty- (or age-) related bio-physiological alterations in conjunction with gender (e.g., sex hormones) might influence disease severity of NAFLD. The exact mechanisms underlying the observed associations and potential gender interactions remain unclear. A better understanding of how gender and reproductive lifecycle (or sex hormones) impact NAFLD disease severity is critical for developing a tailored approach to prevention and treatment of this emerging condition.

\section{Acknowledgments}

Source of funding: The Nonalcoholic Steatohepatitis Clinical Research Network (NASH CRN) is supported by the National Institute of Diabetes and Digestive and Kidney Diseases (NIDDK) (grants U01DK061718, U01DK061728, U01DK061731, U01DK061732, U01DK061734, U01DK061737, U01DK061738, U01DK061730, U01DK061713), and the National Institute of Child Health and Human Development (NICHD). Several clinical centers use support from General Clinical Research Centers or Clinical and Translational Science Awards in conduct of NASH CRN Studies (grants UL1RR024989, M01RR000750, M01RR00188, UL1RR02413101, M01RR000827, UL1RR02501401, M01RR000065, M01RR020359). Dr. Manal Abdelmalek is supported by a NIH/NIDDK K23 Career Development Award (K23-DK062116).

The authors would like to thank Dr. Yuliya Lokhnygina, Department of Biostatistics, Duke University, Durham, $\mathrm{NC}$, for her critical consideration and insight regarding statistical approach used in this analysis and her assistance in statistical analysis. This publication was made possible by Grant Number UL1RR024128 from the National Center for Research Resources (NCRR), a component of the National Institutes of Health (NIH), and NIH Roadmap for Medical Research. Its contents are solely the responsibility of the authors and do not necessarily represent the official view of NCRR or NIH.

\section{Abbreviations}

$\begin{array}{ll}\text { ANOVA } & \text { analysis of variance } \\ \text { BMI } & \text { body mass index } \\ \text { COR } & \text { cumulative odds ratio } \\ \text { HDL } & \text { high-density lipoprotein } \\ \text { IGF } & \text { impaired fasting glucose } \\ \text { LDL } & \text { low-density lipoprotein }\end{array}$


MET

NASH

NASH CRN

SD

SH

US

ALT

AST

GGT

QUICKI metabolic equivalent tasks, NAFLD, nonalcoholic fatty liver disease

Nonalcoholic Steatohepatitis

Nonalcoholic Steatohepatitis Clinical Research Network

standard deviation

steatohepatitis

United States

alanine transaminase

aspartate transaminase

gamma-glutamyl transpeptidase

quantitative insulin sensitivity check index

\section{References}

1. Angulo P. Nonalcoholic fatty liver disease. New England Journal of Medicine. 2002; 346:1221-31. [PubMed: 11961152]

2. Schwimmer JB, Deutsch R, Kahen T, Lavine JE, Stanley C, Behling C. Prevalence of fatty liver in children and adolescents. Pediatrics. 2006; 118:1388-93. [PubMed: 17015527]

3. Feldstein AE, Charatcharoenwitthaya P, Treeprasertsuk S, Benson JT, Enders FB, Angulo P. The natural history of non-alcoholic fatty liver disease in children: a follow-up study for up to 20 years. Gut. 2009; 58:1538-44. [PubMed: 19625277]

4. Carter-Kent C, Yerian LM, Brunt EM, Angulo P, Kohli R, Ling SC, Xanthakos SA, Whitington PF, Charatcharoenwitthaya P, Yap J, Lopez R, McCullough AJ, Feldstein AE. Nonalcoholic steatohepatitis in children: a multicenter clinicopathological study. Hepatology. 2009; 50:1113-20. [PubMed: 19637190]

5. Schwimmer JB, Behling C, Newbury R, Deutsch R, Nievergelt C, Schork NJ, Lavine JE. Histopathology of pediatric nonalcoholic fatty liver disease. Hepatology. 2005; 42:641-9. [PubMed: 16116629]

6. Suzuki A, Abdelmalek MF. Nonalcoholic fatty liver disease in women. Womens Health (Lond Engl). 2009; 5:191-203. [PubMed: 19245356]

7. Suzuki A, Angulo P, Lymp J, St Sauver J, Muto A, Okada T, Lindor K. Chronological development of elevated aminotransferases in a nonalcoholic population. Hepatology. 2005; 41:64-71. [PubMed: 15690483]

8. Clark JM, Brancati FL, Diehl AM. Nonalcoholic fatty liver disease. Gastroenterology. 2002; 122:1649-57. [PubMed: 12016429]

9. Suzuki A, Abdelmalek MF, Unalp-Arida A, Yates K, Sanyal A, Guy C, Diehl AM. Regional anthropometric measures and hepatic fibrosis in patients with nonalcoholic Fatty liver disease. Clin Gastroenterol Hepatol. 2010; 8:1062-9. [PubMed: 20728571]

10. Codes L, Asselah T, Cazals-Hatem D, Tubach F, Vidaud D, Parana R, Bedossa P, Valla D, Marcellin P. Liver fibrosis in women with chronic hepatitis C: evidence for the negative role of the menopause and steatosis and the potential benefit of hormone replacement therapy. Gut. 2007; 56:390-5. [PubMed: 17005762]

11. Patton HM, Sirlin C, Behling C, Middleton M, Schwimmer JB, Lavine JE. Pediatric nonalcoholic fatty liver disease: a critical appraisal of current data and implications for future research. J Pediatr Gastroenterol Nutr. 2006; 43:413-27. [PubMed: 17033514]

12. Patton HM, Lavine JE, Van Natta ML, Schwimmer JB, Kleiner D, Molleston J. Clinical correlates of histopathology in pediatric nonalcoholic steatohepatitis. Gastroenterology. 2008; 135:19611971. e2. [PubMed: 19013463]

13. Andersen O, Pedersen SB, Svenstrup B, Hansen BR, Paulsen SK, Rathje GS, Richelsen B, Nielsen JO, Madsbad S, Iversen J, Haugaard SB. Circulating sex hormones and gene expression of 
subcutaneous adipose tissue oestrogen and alpha-adrenergic receptors in HIV-lipodystrophy: implications for fat distribution. Clin Endocrinol (Oxf). 2007; 67:250-8. [PubMed: 17524033]

14. Goran MI, Gower BA. Longitudinal study on pubertal insulin resistance. Diabetes. 2001; 50:244450. [PubMed: 11679420]

15. Nonalcoholic steatohepatitis clinical research network. Hepatology. 2003; 37:244. [PubMed: 12540771]

16. Lavine JE, Schwimmer JB, Molleston JP, Scheimann AO, Murray KF, Abrams SH, Rosenthal P, Sanyal AJ, Robuck PR, Brunt EM, Unalp A, Tonascia J. Treatment of nonalcoholic fatty liver disease in children: TONIC trial design. Contemp Clin Trials. 31:62-70. [PubMed: 19761871]

17. Kleiner DE, Brunt EM, Van Natta M, Behling C, Contos MJ, Cummings OW, Ferrell LD, Liu YC, Torbenson MS, Unalp-Arida A, Yeh M, McCullough AJ, Sanyal AJ. Design and validation of a histological scoring system for nonalcoholic fatty liver disease. Hepatology. 2005; 41:1313-21. [PubMed: 15915461]

18. Brunt EM, Kleiner DE, Wilson LA, Unalp A, Behling CE, Lavine JE, Neuschwander-Tetri BA. Portal chronic inflammation in nonalcoholic fatty liver disease (NAFLD): a histologic marker of advanced NAFLD-Clinicopathologic correlations from the nonalcoholic steatohepatitis clinical research network. Hepatology. 2009; 49:809-20. [PubMed: 19142989]

19. Marshall WA, Tanner JM. Variations in pattern of pubertal changes in girls. Arch Dis Child. 1969; 44:291-303. [PubMed: 5785179]

20. Marshall WA, Tanner JM. Variations in the pattern of pubertal changes in boys. Arch Dis Child. 1970; 45:13-23. [PubMed: 5440182]

21. Block G, Hartman AM, Dresser CM, Carroll MD, Gannon J, Gardner L. A data-based approach to diet questionnaire design and testing. Am J Epidemiol. 1986; 124:453-69. [PubMed: 3740045]

22. Frisancho, AR. Anthropometric Standards: An Interactive Nutritional Reference of Body Size and Body Composition for Children and Adults. Univ Michigan Press; 2008.

23. Kozlov AV, Duvigneau JC, Hyatt TC, Raju R, Behling T, Hartl RT, Staniek K, Miller I, Gregor W, Redl H, Chaudry IH. Effect of estrogen on mitochondrial function and intracellular stress markers in rat liver and kidney following trauma-hemorrhagic shock and prolonged hypotension. Mol Med. 16:254-61. [PubMed: 20379612]

24. Hanada S, Snider NT, Brunt EM, Hollenberg PF, Omary MB. Gender dimorphic formation of mouse Mallory-Denk bodies and the role of xenobiotic metabolism and oxidative stress. Gastroenterology. 138:1607-17. [PubMed: 20064513]

25. Yasuda M, Shimizu I, Shiba M, Ito S. Suppressive effects of estradiol on dimethylnitrosamineinduced fibrosis of the liver in rats. Hepatology. 1999; 29:719-27. [PubMed: 10051473]

26. Janfaza M, Sherman TI, Larmore KA, Brown-Dawson J, Klein KO. Estradiol levels and secretory dynamics in normal girls and boys as determined by an ultrasensitive bioassay: a 10 year experience. J Pediatr Endocrinol Metab. 2006; 19:901-9. [PubMed: 16995570]

27. Shirtcliff EA, Dahl RE, Pollak SD. Pubertal development: correspondence between hormonal and physical development. Child Dev. 2009; 80:327-37. [PubMed: 19466995]

28. Biro FM, Lucky AW, Simbartl LA, Barton BA, Daniels SR, Striegel-Moore R, Kronsberg SS, Morrison JA. Pubertal maturation in girls and the relationship to anthropometric changes: pathways through puberty. J Pediatr. 2003; 142:643-6. [PubMed: 12838192]

\section{Members of the Nonalcoholic Steatohepatitis Clinical Research Network Clinical Centers}

\section{Case Western Reserve University Clinical Centers}

- MetroHealth Medical Center, Cleveland, OH: Arthur J. McCullough, MD; Patricia Brandt; Srinivasan Dasarathy, MD; Jaividhya Dasarathy, MD; Carol Hawkins, RN; Yao-Chang Liu, MD (2004-2009); 
- Cleveland Clinic Foundation, Cleveland, OH: Arthur J. McCullough, MD; Srinivasan Dasarathy, MD; Mangesh Pagadala, MD; Ruth Sargent, LPN; Lisa Yerian, MD; Claudia Zein, MD

California Pacific Medical Center: Raphael Merriman, MD; Anthony Nguyen

Duke University Medical Center, Durham, NC: Manal F. Abdelmalek, MD, MPH; Stephanie Buie; Anna Mae Diehl, MD; Marcia Gottfried, MD (2004-2008); Cynthia D Guy, MD; Meryt Hanna; Paul Killenberg, MD (2004-2008); Samantha Kwan, MS (2006-2009);

Yi-Ping Pan; Dawn Piercy, FNP; Melissa Smith

Indiana University School of Medicine, Indianapolis, IN: Elizabeth Byam, RN; Naga Chalasani, MD; Oscar W. Cummings, MD; Ann Klipsch, RN; Jean P. Molleston, MD; Linda Ragozzino, RN; Girish Subbarao, MD; Raj Vuppalanchi, MD

Johns Hopkins Hospital, Baltimore, MD: Michael Torbenson, MD; Ann Scheimann MD, MBA; Kim Pfeiffer RN

Northwestern University Feinberg School of Medicine/Children's Memorial Hospital: Mark H. Fishbein, MD; Katie Jacques; Ann Quinn, RD; Cindy Riazi, RN; Peter F. Whitington, MD

Saint Louis University, St Louis, MO/Texas Children's Hospita, Houston, TX: Sarah Barlow, MD (2002-2007); Jose Derdoy, MD; Joyce Hoffmann; Debra King, RN; Andrea Morris; Joan Siegner, RN; Susan Stewart, RN; Brent A. Neuschwander-Tetri, MD; Judy Thompson, RN; Stephanie Abrams MD; Leanel Fairly RN

University of California San Diego, San Diego, CA: Cynthia E Behling, MD, PhD; Janis Durelle; Joel E. Lavine, MD, PhD; Rohit Loomba, MD; Anya Morgan; Heather Patton, MD; Jeffrey B. Schwimmer, MD

University of California San Francisco, San Francisco, CA: ; Nathan M. Bass, MD, PhD; Linda D. Ferrell, MD; Danuta Filipowski, MD; Raphael Merriman, MD; Mark Pabst; Philip Rosenthal, MD; University of Washington Medical Center, Seattle, WA: Matthew Yeh, $\mathrm{MD}, \mathrm{PhD}$

Virginia Commonwealth University, Richmond, VA: Sherry Boyett, RN, BSN; Melissa J. Contos, MD; Michael Fuchs, MD; Amy Jones; Velimir AC Luketic, MD; Puneet Puri, MD;; Arun J. Sanyal, MD; Carol Sargeant, RN, BSN, MPH; Kimberly Noble;

Virginia Mason Medical Center ${ }^{1}$, Seattle, WA: Kris V. Kowdley, MD; Jody Mooney, MS; James Nelson, PhD; Sarah Ackermann; Cheryl Saunders, MPH; Vy Trinh; Chia Wang, MD

Washington University, St. Louis, MO: Elizabeth M. Brunt, MD

\section{Resource Centers}

National Cancer Institute, Bethesda, MD: David E. Kleiner, MD, PhD

\footnotetext{
$1_{\text {original grant with University of Washington }}$
} 
National Institute of Diabetes and Digestive and Kidney Diseases, Bethesda, MD: Edward C. Doo, MD; Jay H. Hoofnagle, MD; Patricia R. Robuck, PhD, MPH (Project Scientist)

Johns Hopkins University, Bloomberg School of Public Health (Data Coordinating Center), Baltimore, MD: Patricia Belt, BS; Jeanne M. Clark, MD, MPH; Ryan Colvin, MPH; Michele Donithan, MHS; Mika Green, MA; Milana Isaacson, BS; Wana Kim, BS; Laura Miriel; Alice Sternberg, ScM; James Tonascia, PhD; Aynur Ünalp, MD, PhD; Mark Van Natta, MHS; Ivana Vaughn, MPH; Laura Wilson, ScM; Katherine Yates, ScM 
Steatosis $(p=0.007)$
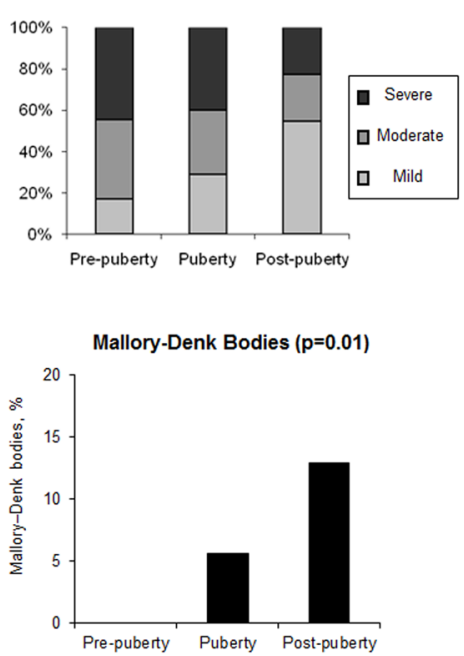

Steatohepatitis $(p=0.005)$
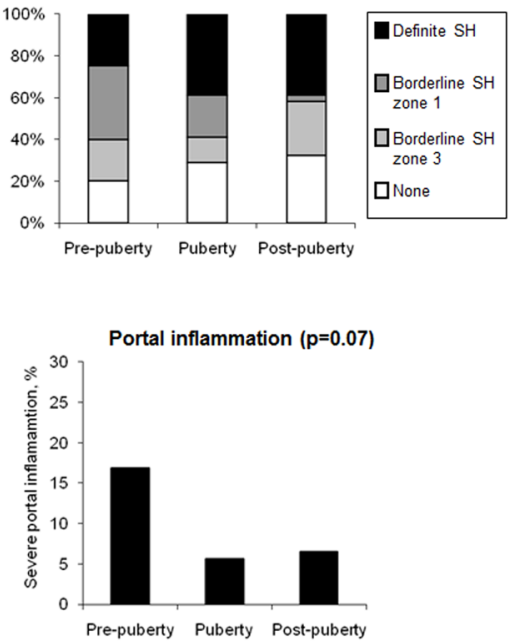

Figure 1. Associations of Pubertal Development with Steatosis, Steatohepatitis, Mallory-Denk bodies, and Portal Inflammation in Total Population

Figures show the associations of pubertal stages (horizontal axis) with the prevalence of up to mild (grade $0-1$ ), moderate (grade 2) and severe (grade 3) steatosis (left upper), the prevalence of none (simple steatosis), borderline steatohepatitis zone 3 (or adult pattern), borderline steatohepatitis zone 1(pediatric pattern), and definite steatohepatitis (right upper), the prevalence of severe (grade 2) portal inflammation (left lower), and the prevalence of Mallory-Denk bodies (right lower). P-values are from exact tests. SH: steatohepatitis 
Table 1

Tanner Stages for Boys and Girls 19, 20

\begin{tabular}{|c|c|c|c|}
\hline Tanner stage & Pubic hair (boys and girls) & Genitals (boys) & Breasts (Girls) \\
\hline 1 & no pubic hair & $\begin{array}{l}\text { testicular volume }<1.5 \mathrm{ml} \text {; small penis of } \\
3 \mathrm{~cm} \text { or less }\end{array}$ & $\begin{array}{l}\text { no glandular tissue; areola follows the skin } \\
\text { contours of the chest }\end{array}$ \\
\hline 2 & $\begin{array}{l}\text { small amount of long, downy } \\
\text { hair with slight pigmentation at } \\
\text { the base of the penis and } \\
\text { scrotum (males) or on the labia } \\
\text { majora (females) }\end{array}$ & $\begin{array}{l}\text { testicular volume }(1.6-6 \mathrm{ml}) ; \text { skin on } \\
\text { scrotum thins, reddens and enlarges; penis } \\
\text { length unchanged }\end{array}$ & $\begin{array}{l}\text { breast bud forms, with small area of } \\
\text { surrounding glandular tissue; areola begins to } \\
\text { widen }\end{array}$ \\
\hline 3 & $\begin{array}{l}\text { more coarse and curly hair, } \\
\text { extending laterally }\end{array}$ & $\begin{array}{l}\text { testicular volume }(6-12 \mathrm{ml}) \text {; scrotum } \\
\text { enlarges further; penis begins to lengthen } \\
\text { to about } 6 \mathrm{~cm}\end{array}$ & $\begin{array}{l}\text { breast begins to become more elevated, and } \\
\text { extends beyond the borders of the areola, } \\
\text { which continues to widen but remains in } \\
\text { contour with surrounding breast }\end{array}$ \\
\hline 4 & $\begin{array}{l}\text { adult-like hair quality, } \\
\text { extending across pubis but } \\
\text { sparing medial thighs }\end{array}$ & $\begin{array}{l}\text { testicular volume }(12-20 \mathrm{ml}) \text {; scrotum } \\
\text { enlarges further and darkens; penis } \\
\text { increases in length to } 10 \mathrm{~cm} \text { and } \\
\text { circumference }\end{array}$ & $\begin{array}{l}\text { increased breast size and elevation; areola } \\
\text { and papilla form a secondary mound } \\
\text { projecting from the contour of the } \\
\text { surrounding breast }\end{array}$ \\
\hline 5 & $\begin{array}{l}\text { hair extending to medial } \\
\text { surface of the thighs }\end{array}$ & $\begin{array}{l}\text { testicular volume }>20 \mathrm{ml} \text {; adult scrotum } \\
\text { and penis of } 15 \mathrm{~cm} \text { in length }\end{array}$ & $\begin{array}{l}\text { breast reaches final adult size; areola returns } \\
\text { to contour of the surrounding breast, with a } \\
\text { projecting central papilla }\end{array}$ \\
\hline
\end{tabular}




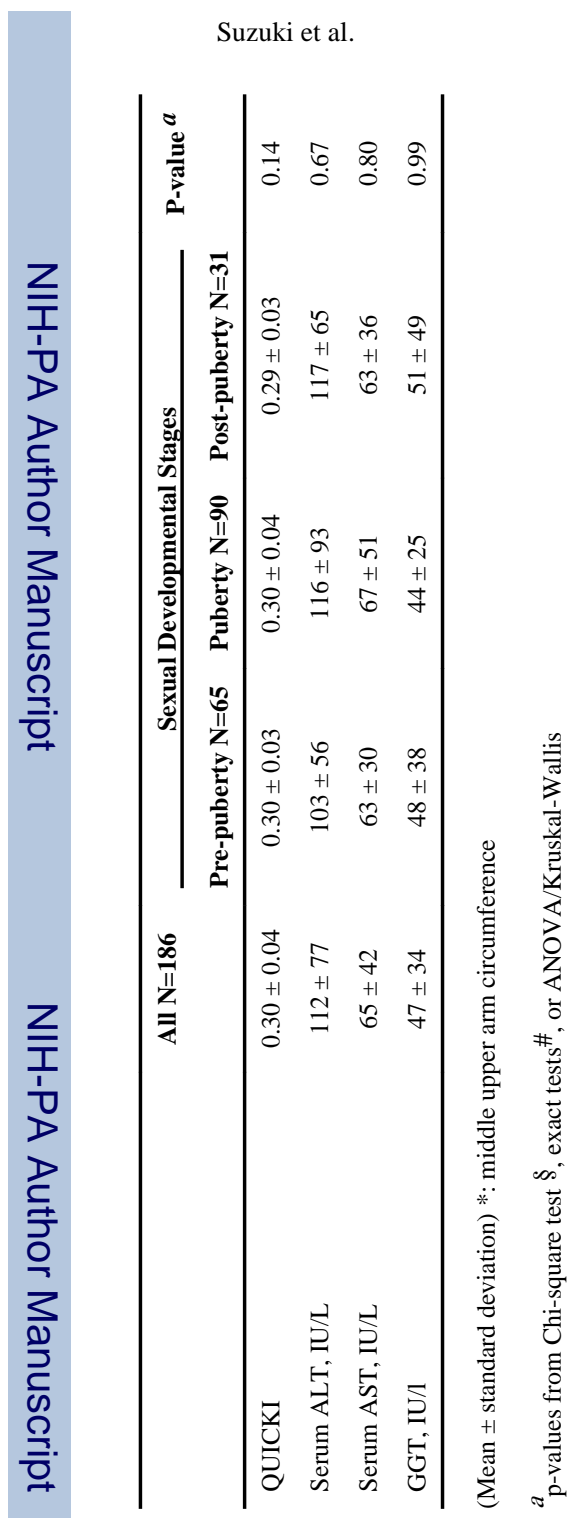

Page 16 


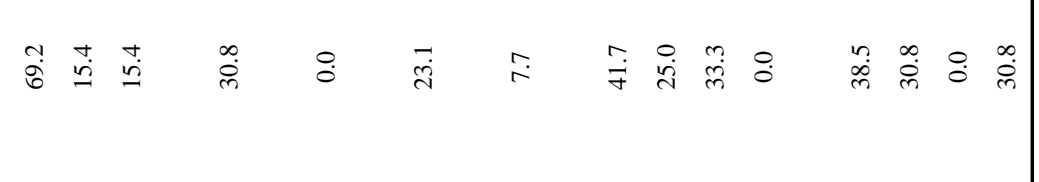

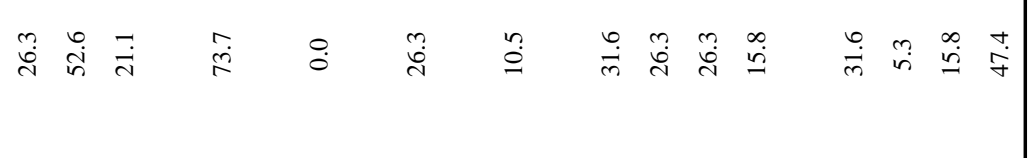

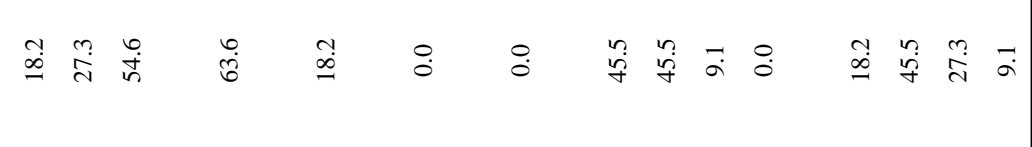

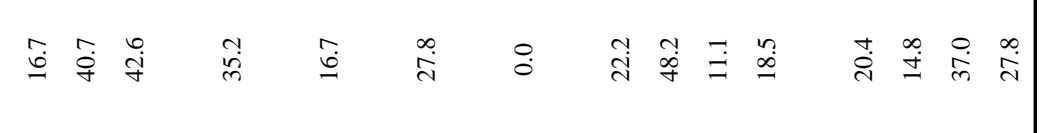
$\stackrel{5}{\circ} \stackrel{5}{\circ} \stackrel{\frac{0}{0}}{8}$ 


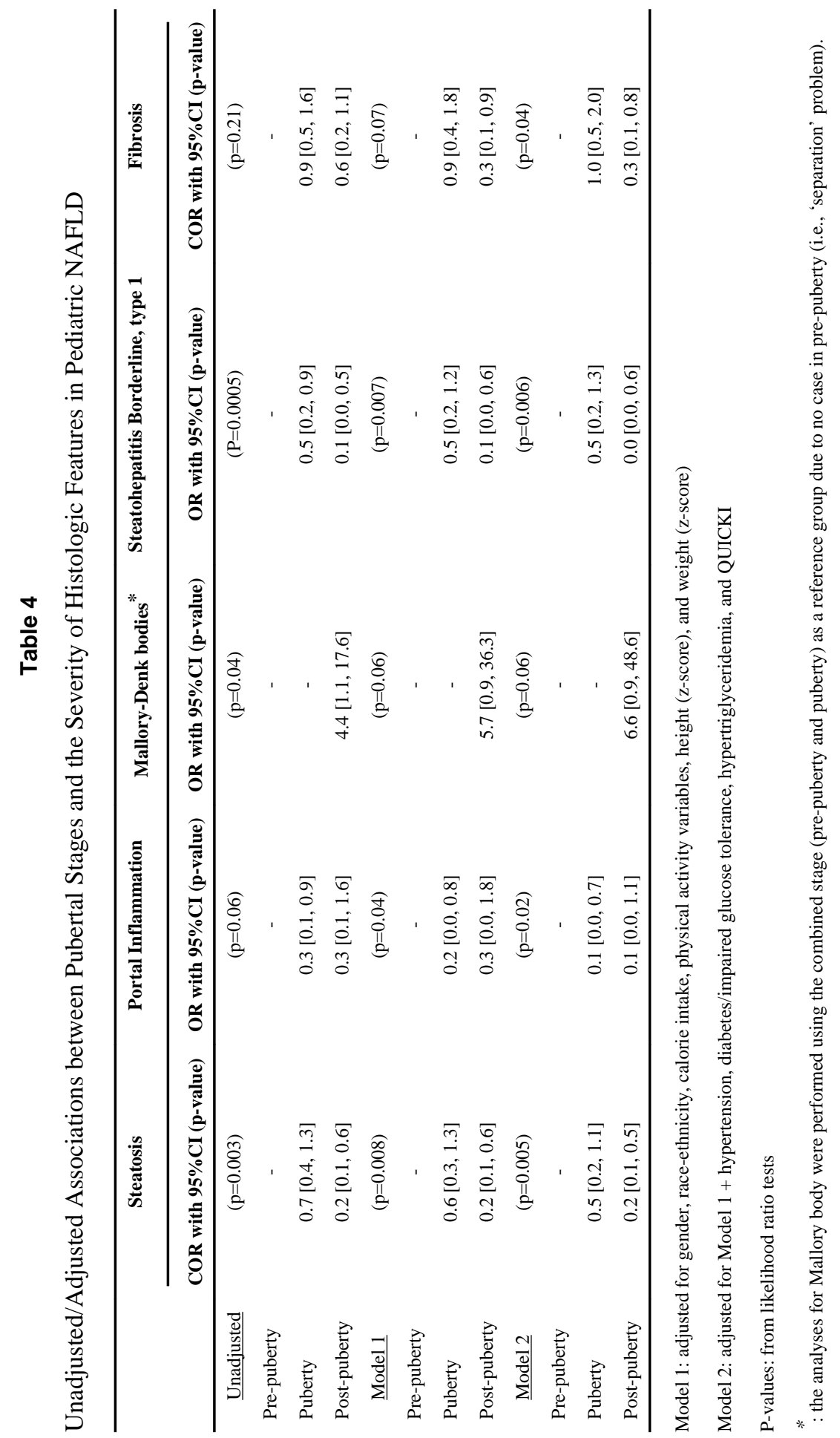

